\title{
Ileana and Hades: a successful adventure into a large collaboration
}

\section{Paolo Finocchiaro ${ }^{1}$}

Istituto Nazionale di Fisica Nucleare - Laboratori Nazionali del Sud

Via S.Sofia 62, 95125 Catania, Italy

E-mail: finocchiaroelns.infn.it

Ileana Iori worked in the HADES collaboration during 1995-2008, thus spending there a relevant part of her scientific life. As leader of the Milan group, as well as outstanding member of the italian participation, she represented a reference point both in the every day life of the experiments and, at a more political level, in the Collaboration Board. The initial challenge of building such a high-performance spectrometer was finally won, as testified by the currently increasing number of relevant physics results and papers.

XLVIII International Winter Meeting on Nuclear Physics - BORMIO2010

Bormio, Italy

January 25-29 2010

$1 \quad$ Speaker 


\section{Introduction}

In this paper I will outline the development of the HADES spectrometer, along with the results of the first experiments, highlighting the perspective and contribution of the italian part of the collaboration where Ileana Iori belonged. Therefore I will start by shortly describing the physical background that led to the proposal of HADES, then moving on with the chronology of its construction, test, commissioning, and concluding with some experiments and results.

\section{Physical motivations for HADES}

In order to better clarify where in the nuclear physics landscape the HADES physics is located, I reported in Figure 1 a rough pictorial summary of the basic phenomena and interpretations as related to the energy range of the collisions used to study them.

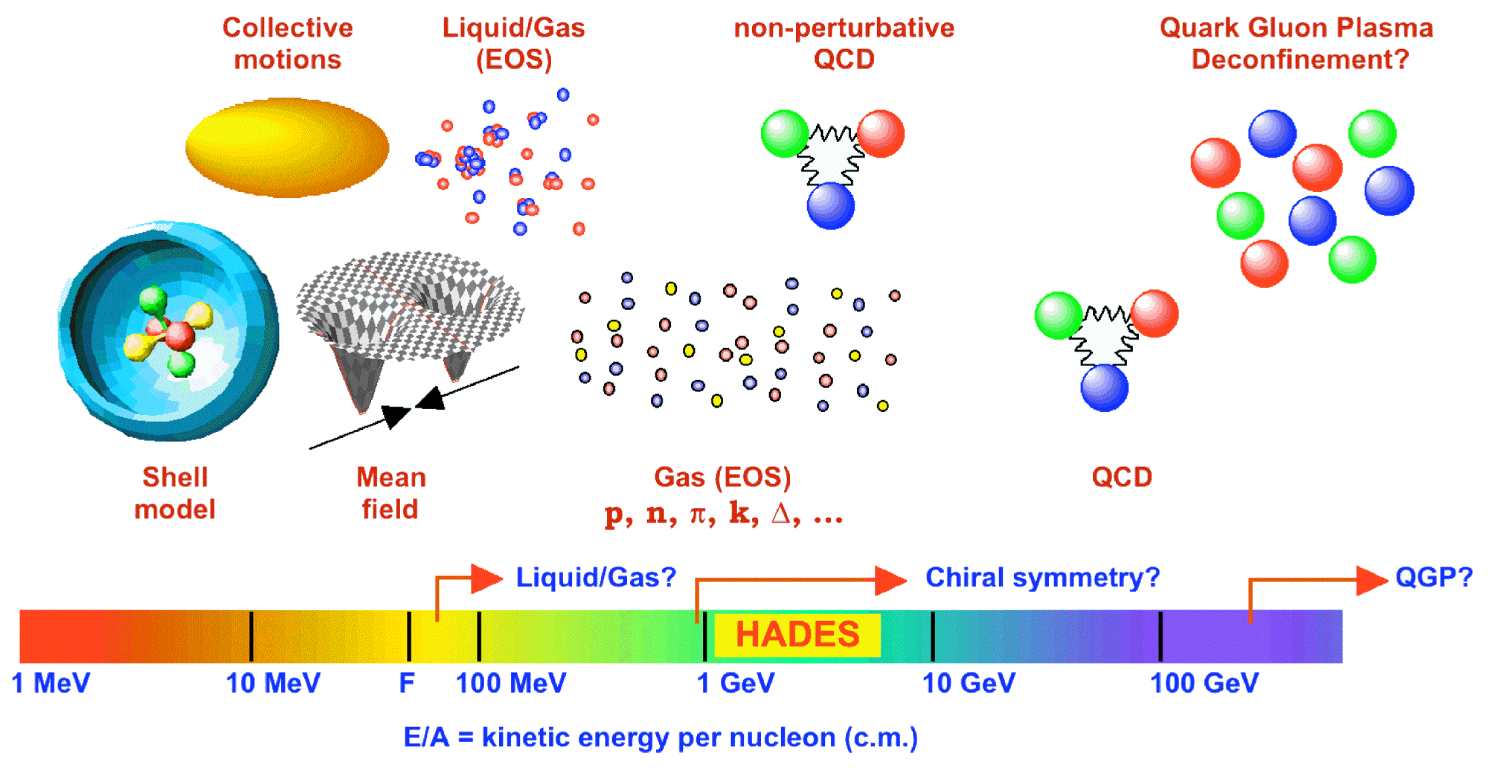

Figure 1. Pictorial view of the HADES placement within the nuclear physics landscape.

A little bit of history shows that the initial question was, and still is, about the behavior and properties of the nuclear matter at high temperature and/or high density. In particular people were wondering if there might be hints of chiral symmetry restoration already in the $1 \mathrm{~A} \mathrm{GeV}$ energy range. In such a case the in-medium properties of nuclear matter should change, and the question became "how can we test it?". Several models trying to describe such changes were developed, as for instance in [1-5]. One of the basic questions was whether the vector meson masses in dense matter stay constant or rather they change, and in such a case the new question was whether they simply shifted downward, upward or broadened. Apart from theoretical discussions, the only way to solve the debate was to perform experiments.

How could one test the in-medium properties of nuclear matter? Well, what was needed was a special probe not sensitive to the final state interaction, and a perfect candidate seemed to be the di-electron pairs. Indeed the vector mesons have a decay channel into e+e- pairs, the 
only drawback being the faint branching ratio typically of the order of $10^{-5}$. Pioneering experiments were performed at Bevalac by the DLS collaboration at the end of the ' $80 \mathrm{~s}$, ending up in an unexplained excess in the e+e-production in $\mathrm{C}+\mathrm{C}$ and $\mathrm{Ca}+\mathrm{Ca}$ collisions at $1 \mathrm{~A} \mathrm{GeV}$ with respect to the theoretical expectation [6]. Such an excess was not present in the elementary $\mathrm{p}+\mathrm{p}$ collisions, nor was it found in $\mathrm{p}+\mathrm{A}$ collisions. Many people throughout the years did not believe in these data, until HADES unequivocally confirmed them. A similar excess was also observed at CERN in the CERES and NA45 experiments, but as they had been performed at a much higher energy, there were possible alternative explanations for it.

Meanwhile, in the early ' $90 \mathrm{~s}$, the DLS spectrometer was dismissed, and then there was no way to solve the so-called DLS puzzle in short time.

\section{The birth of HADES}

In 1994 a collaboration was formed, centered at GSI Darmstadt, with the aim of studying the in-medium properties of nuclear matter and mesons at beam energies of $1-2 \mathrm{~A} \mathrm{GeV}$, by means of the e+e- decay channel. In order to pursue these studies the collaboration planned to build a powerful yet challenging spectrometer, whose name was HADES from High Acceptance Di-Electron Spectrometer. In Figure 2 we can see a sketch of the spectrometer as it was originally proposed. As to its features, listed while moving along the beam direction, we have a segmented solid target (or alternatively a liquid Hydrogen target), a RICH detector, 2 layers of inner Mini Drift Chambers, a toroidal magnet, 2 layers of outer Mini Drift Chambers, a TOF detector, a SHOWER detector, and finaly a high-performance data acquisition and trigger system. The system has an intrinsic hexagonal symmetry, therefore each subsystem is composed of 6 sectors.
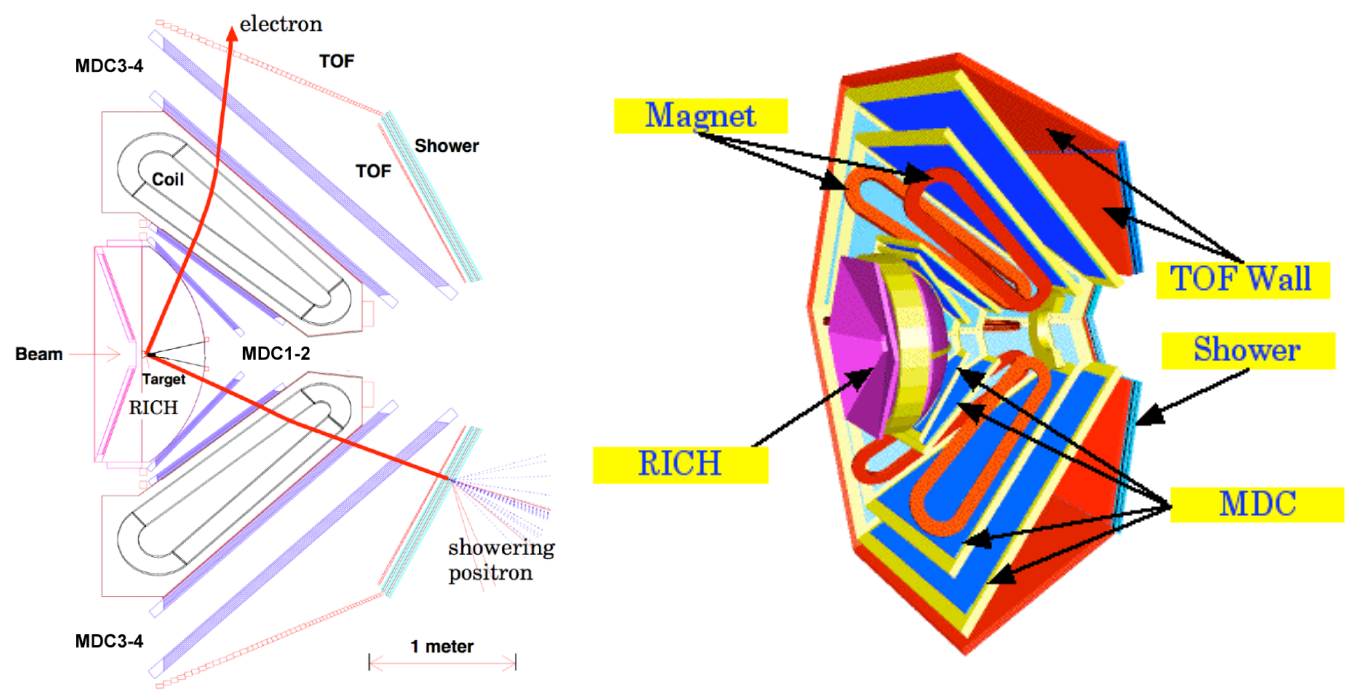

Figure 2. A sketch of the HADES spectrometer as originally proposed; in the righthand side drawing two sectors were removed for better clarity.

The RICH, in principle blind to all particles except to fast electrons, is devoted to the e+echannel selection. The four MDC layers, along with the magnet, perform the high resolution particle tracking. The SHOWER provides a redundant tracking and an additional electron 
trigger at angles between 18 and 45 degrees, whereas the TOF wall does the same at angles between 45 and 88 degrees [7].

\section{The italian group joins the collaboration}

In June 1995, during the HADES collaboration meeting II in Obernai (France), the italian group expressed the intention of joining the collaboration. Lead by Ileana the group, based at INFN Sezione di Milano and INFN Laboratori Nazionali del Sud in Catania, was supposed to take care of constructing the TOF detector, along with groups from Rez (Czech Republic), Bratislava (Slovakia), Moscow (Russia). An application was then submitted to INFN, but only a small fund was granted by INFN to its two groups for 1996, in order to keep in touch with the collaboration: no detector was funded.

By December 1995, during the Hades collaboration meeting III at GSI, a decision was made to start some TOF tests and prototyping, in order to try applying again in summer 1996 for 1997. Meanwhile Ileana decided that the leadership of the TOF group would better move to LNS Catania.

In the original plan the TOF wall was supposed to cover the whole angular acceptance but, in order to make the proposal more realistic as to financial and manpower requirements, it was decided to apply only for the backward part and to cover the 18-45 degrees region with 24 large slabs of plastic scintillators, called TOFINO, to be built by a russian group. As for the TOF, the Milan group would develop the front-end digital electronics (ADC's,TDC's, shapers, active delays), whereas the Catania group would take care of the detector itself (scintillators, photomultipliers, voltage dividers, discriminators).

In July 1996 the new application for funding was submitted, and in September it was approved. As a consequence INFN became formally member of the HADES collaboration.

In december 1996, during the HADES collaboration meeting $\mathrm{V}$ in Bensheim, the TOF project was officially presented to the collaboration; in particular Ileana's group showed the design of the new 32-channels TDC and ADC for the HADES TOF, whereas the LNS group showed the preliminary results of bench tests with prototype modules of HADES TOF. Decisions were made concerning the number and size of the scintillating rods and lightguides, and the Rez, Bratislava and Moscow groups showed their preliminary results concerning simulations and bench tests on scintillators, PMTs, magnetic shields, power supply systems, mechanical housing and holders.

\section{First beam tests and results}

In April 1997 a preliminary in-beam test was made with an 8-rod TOF module, some spare scintillators and an MDC chamber, followed by a more advanced test in July with one RICH sector, one MDC, half TOF sector, one SHOWER sector. A sketch of the two setups is shown in Figure 3.

The reaction was $\mathrm{U}+\mathrm{Pb}$ at $1 \mathrm{~A} \mathrm{GeV}$, and the overall time of flight spectrum obtained looked very similar to what expected from a $\mathrm{QMD}$ simulation of a $\mathrm{Au}+\mathrm{Au}$ reaction at $1 \mathrm{~A} \mathrm{GeV}$. At the same time three different models of photomultipliers were tested, and this allowed to 
select the best one (EMI 9133B) as the final one to be purchased. A set of plots, summarizing these results is shown in Figure 4.
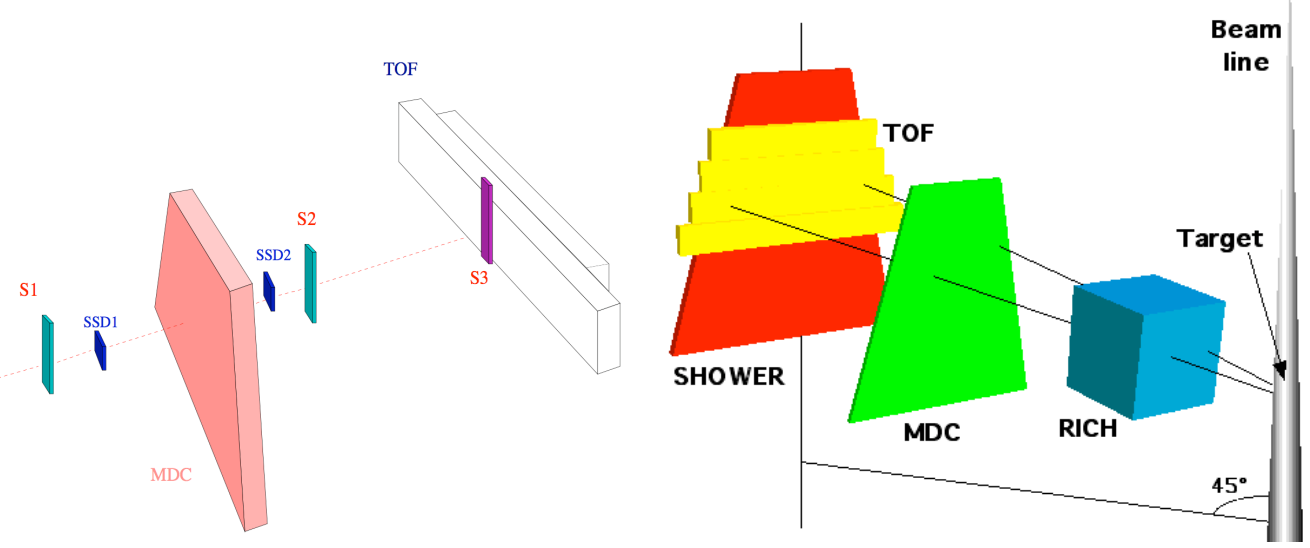

Figure 3. Sketch of the April 1997 (left) and of the July 1997 beam test (right).
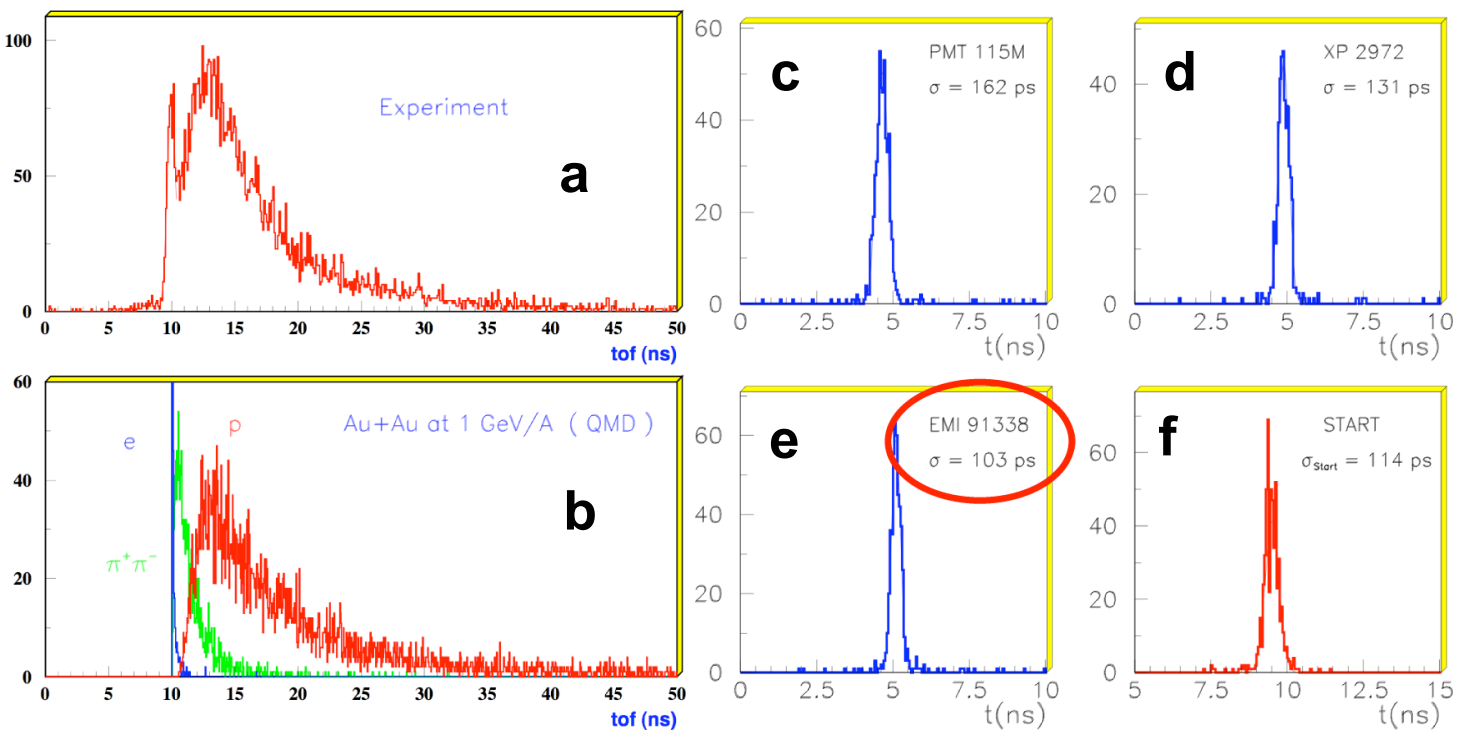

Figure 4. (a) time of flight spectrum for particles produced in a $P b+U$ reaction at $1 \mathrm{~A} G \mathrm{GeV}$. (b) the same spectrum as expected from a $Q M D$ simulation of a $A u+A u$ reaction at $1 \mathrm{~A} \mathrm{GeV}$. (c, $d$, e) time resolution as measured by three different models of photomultipliers, the best one is circled in red. (f) time resolution of the start detector, that was quadratically subtracted to obtain $c, d, e$.

During the second test $\mathrm{e}+\mathrm{e}-$ pairs from gamma conversion were successfully detected and identified using the combined detector prototypes, thus proving that the overall operational scheme foreseen for the spectrometer was indeed correct.

\section{Prototyping year}

The year 1998 was totally devoted to build detector prototypes, along with electronics and mechanics. In particular, concerning the TOF groups, there was an intense activity in Milan that lead to the construction of a working prototype of TDC, in VME standard, featuring 32 
independent channels with 50ps time resolution, $5 \mu$ s conversion time and fast readout by means of chained block transfer (Figure 5 left). At the same time the group completed a prototype of an active delay module, that allowed a full range delay of 400ns still keeping the time jitter well below 50ps. The following year Ileana's group would finalize new prototypes of the ADC, almost twin module of the TDC, and of the related programmable signal shaper in order to match the output signals from PMTs with the ADC inputs. All of these modules successfully fulfilled the foreseen specifications, and therefore their mass production was started.

Meanwhile the other TOF groups finalized the assembling scheme of the scintillator rods, the optical coupling with lightguides and PMTs, the magnetic shields, the mechanical supports (Figure 5 right), the electrical connections, the power supply system. A particular effort was devoted to the development of a software graphical user interface, quite useful for the online monitoring of the whole detector.
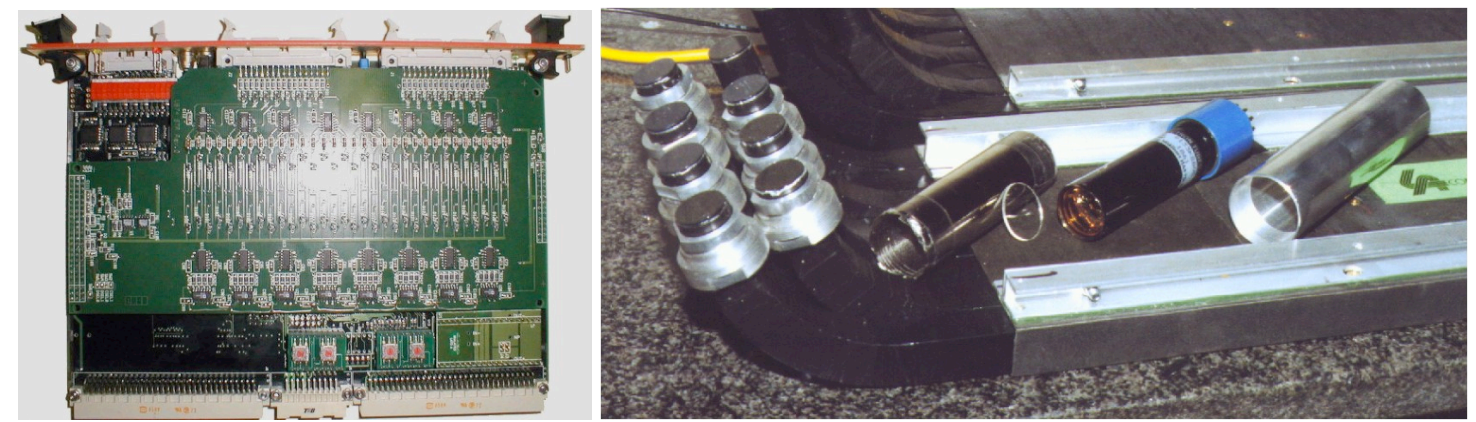

Figure 5. The TDC prototype developed by the Milan group (left); detail of a 8-rod scintillator module, lightguides, optical coupler, PMT, mechanical holder (right).

\section{In-beam prototype commissioning and final installation}

By the end of 1998 a beam test with one complete sector was done (Figure 6 left), followed by other tests in 1999 with two complete sectors (Figure 6 right). These tests allowed to prove that the expected performance of the system was achievable; as a by-product of a test two TOF sectors, together with MDC1, MDC2 and the magnet, allowed to produce the first mass spectrum of the Hades spectrometer, as shown in Figure 7.
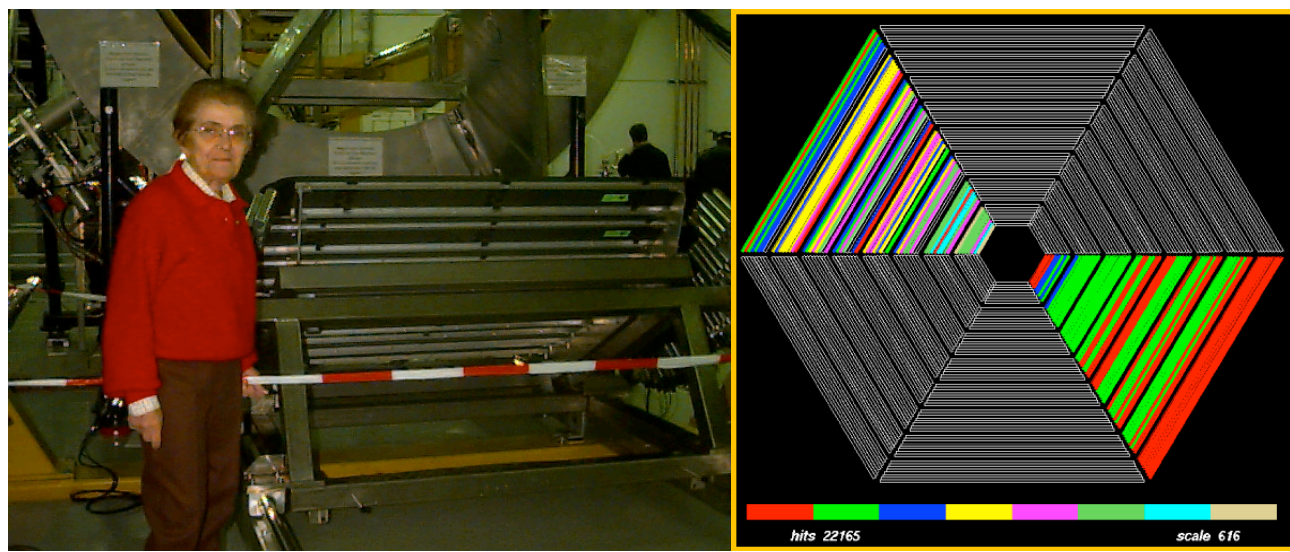

Figure 6. Ileana Iori near the 1-sector TOF detector just before the beam test at GSI in December 1998 (left); online plot of the hit distribution on two TOF sectors during the beam test of June 1999 (right). 
The spectrometer installation went on, and in April 2000 the TOF subsystem was completely installed on its final position, and connected to the slow control system and to the data acquisition system. Another beam test in June 2000 allowed to verify that the spectrometer was clearly capable of performing particle identification and tracking, especially e+e- pairs, and to be safely operated for extended periods as requested for the real physics experiments (Figure 8). Still there were missing parts, namely MDC3, MDC4 and forward TOF, that due to higher complexity together with lack of funds and manpower, had been postponed and would have been completed a few years later. However, even though operated at lower position resolution, by the end of 2001 Hades was ready for a real physics commissioning run that was successfully performed with a $\mathrm{C}+\mathrm{C}$ reaction at $2 \mathrm{~A} \mathrm{GeV}$.

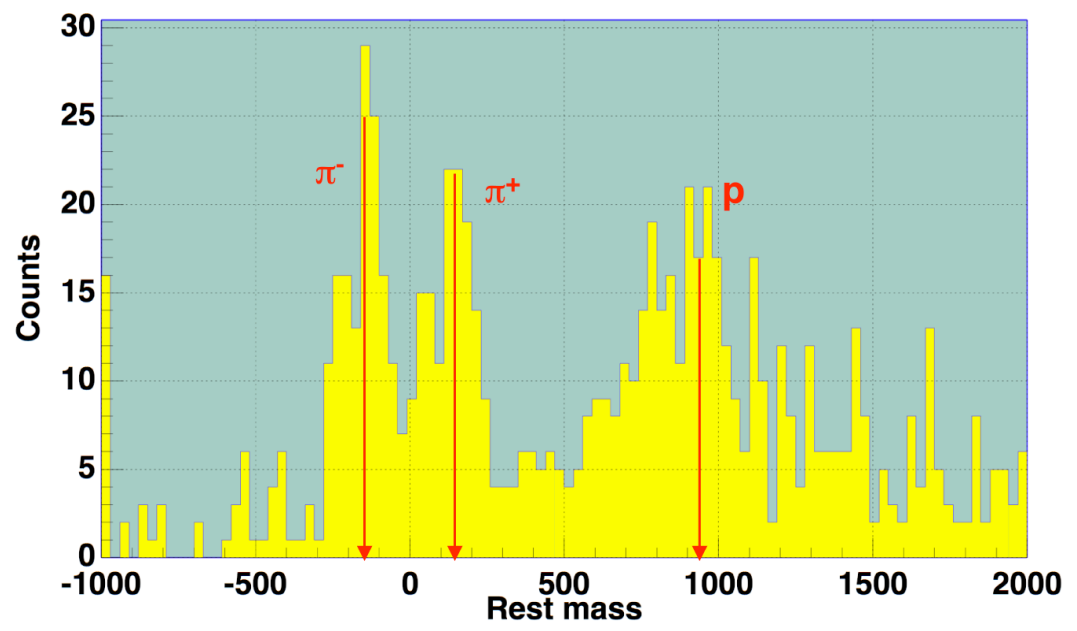

Figure 7. The first (low resolution) mass spectrum of the Hades spectrometer, produced during a beam test in September 1999 using two sectors of TOF, MDC1, MDC2 and the magnet.

\section{The experiments}

After one more year of fine tuning and preparation, the first experiment was done in November 2002 on the $\mathrm{C}+\mathrm{C}$ system at $2 \mathrm{~A} \mathrm{GeV}$. In a few weeks of beam time the experiment collected 213 millions of events, and the complex data analysis needed a few years to be performed. Finally in 2007 these data were published on Physical Review Letters [8] and showed a clear evidence for an e+e- excess at $2 \mathrm{~A} \mathrm{GeV}$ : this was the first step toward the solution of the DLS puzzle.

From then on several experiments were performed, collecting an ever increasing number of events due to improvements in the detector systems but mainly to the progress done with the data acquisition and with the high level digital trigger system, that allowed to greatly improve the performance and the reliability of the whole spectrometer. 420 million events were collected in February 2004 on $\mathrm{p}+\mathrm{p}$ at $2.2 \mathrm{GeV}$, then 650 millions in August 2004 on $\mathrm{C}+\mathrm{C}$ at $1 \mathrm{~A} \mathrm{GeV}, 840$ millions in September 2005 on $\mathrm{Ar}+\mathrm{KCl}$ at $1.765 \mathrm{~A} \mathrm{GeV}, 720$ millions in May 2006 on $\mathrm{p}+\mathrm{p}$ at $1.25 \mathrm{GeV}, 1750$ millions in April 2007 on $\mathrm{p}+\mathrm{p}$ at $3.5 \mathrm{GeV}, 1350$ millions in May $2007 \mathrm{don}+\mathrm{p}$ at $1.25 \mathrm{GeV}, 4400$ millions in September 2008 on $\mathrm{p}+\mathrm{Nb}$ at $3.5 \mathrm{GeV}$. The data analysis is still in progress for most of these experiments, nonetheless the Hades collaboration has already 
published about 130 papers: mostly technical at the beginning, about 60 , then about 70 with physics results after 2003. The basic topics concern dileptons in nucleus + nucleus, $p+p$ and $d+p$ collisions, pion production, eta and omega mesons, kaons, open strangeness barions.

One paper in particular Ileana was proud of, namely ref.[9], consequent to the experiment on $\mathrm{C}+\mathrm{C}$ at $1 \mathrm{~A} \mathrm{GeV}$ done in August 2004, for it confirmed the correctness of the e+e- excess in the DLS data by studying the same reaction at the same energy, thus solving the long standing DLS puzzle. Meanwhile the collaboration has also collected evidence for an explanation of such an excess with respect to the theoretical predictions, and the related data are currently being published.

Unfortunately in 2008 INFN decided to stop funding the italian participation to Hades, and this was more than a disappointment for Ileana after 14 years of common efforts to build the spectrometer and produce high quality physics data. Nevertheless, her work, as well as that of the other italian collaborators, was rewarded by the outstanding physics results achieved while being member of the Hades family.

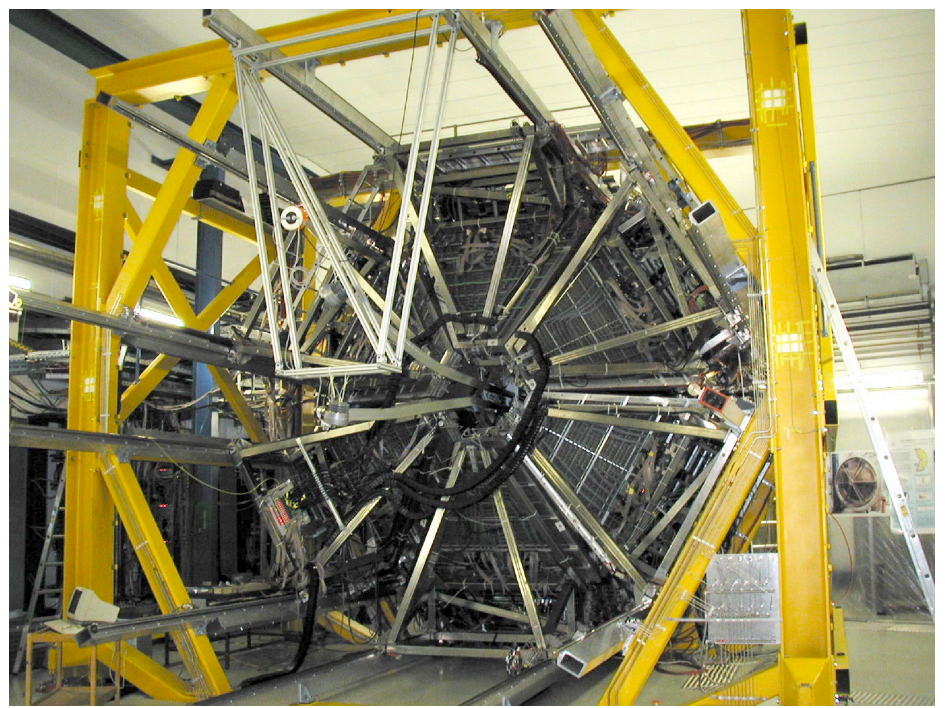

Figure 8. The Hades spectrometer after completing the installation in 2001.

\section{Acknowledgments}

I am grateful to the whole HADES collaboration, previous and current members, who gave Ileana and me the opportunity to live the enthralling adventure of building the spectrometer, operating it and extracting highly significant physics.

I have to explicitly express my gratitude to all those who contributed to make the TOF wall a real piece of working equipment, namely Roberto Bassini, Ciro Boiano, Sergio Brambilla, Augusto Bassi, Massimo Malatesta, Giorgio Bellia, Dima Vasiliev, Titti Agodi, Cettina Maiolino, Paolo Piattelli, Rosa Coniglione, Piera Sapienza, Pavel Tlusty, Andrej Kugler, Vladimir Wagner, Filip Krizek, Radec Pleskac, Stanislav Hlavac, Dusan Zovinec, Martin Benovic, Vladimir Smoliankin. I am indebted with them all. Even though I checked this list carefully I might have forgotten some name and in such a case I apologize. 
I also have to mention here several young italian students in HADES (a few of them are now brilliant scientists) whom Ileana cared for: Laura Fabbietti, Luigi Cosentino, Francesco Eusepi, Stefano Bertino, Alberica Toia, Stefano Spataro, Aldo Bortolotti, Camilla Gilardi, Attilio Tarantola.

A noteworthy support to our participation in HADES came from Antonio Bertin, Emilio Chiavassa, Emilio Migneco, Enzo De Sanctis, Angelo Pagano, Nora De Marco, Alessandro Feliciello.

I must acknowledge here the inestimable support we got from our late genuine friend Helmut Bokemeyer.

And finally I want to thank Ileana Iori for working elbow to elbow with me throughout fourteen years. It was a pleasure and an honor for me to share this long trip with you: thank you, Ileana.

\section{References}

[1] J.J. Sakurai, Currents and Mesons, University of Chicago Press, Chicago (1969)

[2] G.E. Brown, M. Rho, Scaling effective lagrangians in a dense medium, Phys. Rev. Lett. 66 (1991)2720.

[3] G.E. Brown, M. Rho, Chiral restoration in hot matter, Nucl. Phys. A590 (1995)527.

[4] V. Koch, Introduction to Chiral Symmetry, arXiv:nucl-th/9512029v1

[5] U. Mosel, Hadrons in the nuclear medium: introduction and overview, arXiv:nucl-th/9811065v2

[6] R. J. Porter, Dielectron Cross Section Measurements in Nucleus-Nucleus Reactions at 1.0A GeV, Phys. Rev. Lett. 79 (1997)1229.

[7] G.Agakichiev et al., The high-acceptance dielectron spectrometer HADES, Eur. Phys. J. A41, (2009)243.

[8] G.Agakichiev et al., Dielectron production in ${ }^{12} \mathrm{C}+{ }^{12} \mathrm{C}$ collisions at $2 \mathrm{~A} \mathrm{GeV}$ with the HADES spectrometer, Phys. Rev. Lett. 98 (2007)052302.

[9] HADES collaboration, Study of dielectron production in $C+C$ collisions at $1 \mathrm{~A} \mathrm{GeV}$, Physics Letters B663 (2008)43 